\title{
Cosmic inflation and primordial structure
}

\author{
David Wands* \\ Institute of Cosmology and Gravitation, University of Portsmouth \\ E-mail: david.wands@port.ac.uk
}

\begin{abstract}
Cosmic inflation in the very early universe provides a framework in which to understand the seeds of large-scale structure in our Universe. A rapid, accelerated expansion at ultra-high energies can stretch quantum vacuum fluctuations up to extra-galactic scales. I will discuss the impact of recent observations of the cosmic microwave background sky which provide evidence of primordial density perturbations and now, for the first time, possible evidence for primordial gravitational waves as predicted by inflation. I will discuss how inflation compares with alternative models for the origin of structure and how we might further test the physics of inflation through cosmological observations.
\end{abstract}

\section{References}

[1] M. Anselmino, A. Efremov and E. Leader,The theory and phenomenology of polarized deep inelastic scattering, ys. Rept. 261 (1995) 1 [Erratum ibid 281 (1997) 399] [hep-ph/9501369].

[2] R. Penrose and W. Rindler, Spinors and Space-time, Vol. 2: Spinor and twistormethods in space-time geometry, Cambridge University Press, Cambridge U.K.(1986), pg. 501.

Frontiers of Fundamental Physics 14 - FFP14,

15-18 July 2014

Aix Marseille University (AMU) Saint-Charles Campus, Marseille

\footnotetext{
${ }^{*}$ Speaker.
} 